\title{
The Effect of Diabetes-Specific Enteral Nutrition Formula on Cardiometabolic Parameters in Patients with Type 2 Diabetes: A Systematic Review and Meta-Analysis of Randomised Controlled Trials
}

\author{
Omorogieva Ojo ${ }^{1, *(\mathbb{D}}$, Sharon Marie Weldon ${ }^{1,2}$, Trevor Thompson ${ }^{3}$, , Rachel Crockett ${ }^{4}$ and \\ Xiao-Hua Wang ${ }^{5}$ \\ 1 Department of Adult Nursing and Paramedic Science, University of Greenwich, London SE9 2UG, UK \\ 2 Barts Health NHS Trust, The Royal London Hospital, Whitechapel Rd, Whitechapel E1 1BB, UK \\ 3 Department of Psychology, University of Greenwich, London SE10 9LS, UK \\ 4 Division of Psychology, Faculty of Natural Sciences, University of Stirling, Scotland FK9 4LA, UK \\ 5 The School of Nursing, Soochow University, Suzhou 215006, China \\ * Correspondence: o.ojo@greenwich.ac.uk; Tel.: +44-20-8331-8626; Fax: +44-20-8331-8060
}

Received: 24 May 2019; Accepted: 12 August 2019; Published: 15 August 2019

\begin{abstract}
Background: The prevalence of diabetes is on the increase in the UK and worldwide, partly due to unhealthy lifestyles, including poor dietary regimes. Patients with diabetes and other co-morbidities such as stroke, which may affect swallowing ability and lead to malnutrition, could benefit from enteral nutrition, including the standard formula (SF) and diabetes-specific formulas (DSF). However, enteral nutrition presents its challenges due to its effect on glycaemic control and lipid profile. Aim: The aim of this review was to evaluate the effectiveness of diabetes-specific enteral nutrition formula versus SF in managing cardiometabolic parameters in patients with type 2 diabetes. Method: This review was conducted in accordance with the preferred reporting items for systematic reviews and meta-analyses. Three databases (Pubmed, EMBASE, PSYCInfo) and Google scholar were searched for relevant articles from inception to 2 January 2019 based on Population, Intervention, Comparator, Outcomes and Study designs (PICOS) framework. Key words, Medical Subject Heading $(\mathrm{MeSH})$ terms, and Boolean operators (AND/OR) formed part of the search strategy. Articles were evaluated for quality and risks of bias. Results: Fourteen articles were included in the systematic review and five articles were selected for the meta-analysis. Based on the findings of the review and meta-analysis, two distinct areas were evident: the effect of DSF on blood glucose parameters and the effect of DSF on lipid profile. All fourteen studies included in the systematic review showed that DSF was effective in lowering blood glucose parameters in patients with type 2 diabetes compared with SF. The results of the meta-analysis confirmed the findings of the systematic review with respect to the fasting blood glucose, which was significantly lower $(p=0.01)$ in the DSF group compared to SF, with a mean difference of -1.15 (95\% CI $-2.07,-0.23)$ and glycated haemoglobin, which was significantly lower $(p=0.005)$ in the DSF group compared to the SF group following meta-analysis and sensitivity analysis. However, in relation to the sensitivity analysis for the fasting blood glucose, differences were not significant between the two groups when some of the studies were removed. Based on the systematic review, the outcomes of the studies selected to evaluate the effect of DSF on lipid profile were variable. Following the meta-analysis, no significant differences $(p>0.05)$ were found between the DSF and SF groups with respect to total cholesterol, LDL cholesterol and triglyceride. The level of the HDL cholesterol was significantly higher $(p=0.04)$ in the DSF group compared to the SF group after the intervention, with a mean difference of 0.09 (95\% CI, 0.00, 0.18), although this was not consistent based on the sensitivity analysis. The presence of low glycaemic index (GI) carbohydrate, the lower amount of carbohydrate and the higher protein, the presence of mono-unsaturated fatty acids and the different amounts and types of fibre in the DSF compared with
\end{abstract}


SF may be responsible for the observed differences in cardiometabolic parameters in both groups. Conclusion: The results provide evidence to suggest that DSF is effective in controlling fasting blood glucose and glycated haemoglobin and in increasing HDL cholesterol, but has no significant effect on other lipid parameters. However, our confidence in these findings would be increased by additional data from further studies.

Keywords: diabetes specific formula; standard formula; type 2 diabetes; enteral nutrition; enteral tube feeding; lipids; fasting blood glucose; glycated haemoglobin

\section{Introduction}

Diabetes is a metabolic condition which is characterised by chronic hyperglycaemia and is caused by a range of factors including genetic inheritance and environmental influences [1]. The prevalence of diabetes is on the increase in the UK and worldwide, partly due to the changes in lifestyle, including lack of physical activity and unhealthy diets, which lead to overweight and obesity [2-5]. In addition, improvements in technology and the greater awareness of the condition have meant that diabetes is now better detected and more people are engaging in screening programmes. About $90 \%$ of patients with diabetes are diagnosed with type 2 diabetes [6-8]. The impact of diabetes on the people living with the condition can be profound in terms of morbidity and mortality, as well as a cost burden to the National Health Service (NHS). Individuals with diabetes are more likely to be admitted to hospital and it can have a significant effect on the quality of life of patients $[9,10]$. Diabetes is a major risk factor for kidney dysfunction, lower limb amputations, retinopathy, cardiovascular disease and other co-morbidities such as stroke, which can lead to swallowing problems and malnutrition. Based on these issues, diabetes continues to be a major public health concern in the UK and globally, and strategies for managing the condition continue to evolve. Often, management relies on lifestyle modifications such as increased physical activity levels and the use of dietary interventions in order to prevent the onset of type 2 diabetes and ultimately reduce the possibility of diabetic complications [11]. However, in patients who are sedentary and immobile, the use of physical activity as a strategy for managing the condition is sometimes impracticable.

Therefore, individuals with diabetes and other conditions, such as stroke, which could affect mobility and swallowing ability, may benefit from enteral nutrition such as oral nutrition supplements and the use of a nasogastric feeding tube (for short-term feeding) or a percutaneous endoscopic gastrostomy tube for long-term intervention to deliver enteral feeds and formulas [12]. Usually, these individuals have functional guts and the essence is to provide adequate nutrition, hydration and medication to these patients in order to improve their nutritional status and clinical outcomes, including quality of life.

Why it is important to do this review:

The current review focuses mainly on patients with type 2 diabetes from a range of backgrounds, including those attending diabetes centre/outpatient diabetic clinics, rehabilitation departments, ambulatory patients, nursing homes and long-term care facilities, and intensive care units. Patients with diabetes are at a greater risk of developing stroke, peripheral vascular disease, renal impairment and dementia compared with those without the condition due to chronic hyperglycaemia [13]. The long-term complications of diabetes, including its co-morbidities, have implications for the length of hospital stay. Thus, while the average length of hospital stay in patients with diabetes as the primary diagnosis has been estimated to be 4.3 days, it is 8 days in patients with additional diagnoses and 3.1 days in all hospitalisations [14]. The use of enteral feeding in patients with diabetes can present a range of challenges in the control of blood glucose levels and other cardiometabolic parameters [15]. These parameters, including lipid profile, such as total cholesterol, high density lipoprotein cholesterol, low density lipoprotein cholesterol and triglyceride, are important biomarkers in patients with type 2 diabetes as they have implications for insulin resistance and cardiovascular mortality. 
In patients with diabetes who are on enteral nutrition, the enteral feeds provided can be in the form of either Standard Formulas (SF) or Diabetes Specific Formulas (DSF). Enteral feeding formulas have a tendency to promote hyperglycaemia and insulinemic responses in patients with diabetes and in healthy subjects [16,17]. In addition, the effect of enteral nutrition on blood glucose parameters may be due to the fact that continuous enteral feeding is a source of continuous supply of glucose, providing 10-20 g of carbohydrates per hour, which is not the same during normal eating [15]. The absence of the normal postprandial glucose peak in patients with diabetes on enteral nutrition makes the management of hyperglycaemia difficult [15]. On the other hand, the effect of different types and amounts of fibre and mono-unsaturated fatty acids in various enteral feeds may influence lipid profile and other cardiometabolic parameters such as fasting blood glucose and glycated haemoglobin in patients with type 2 diabetes [18]. The role of the different enteral feeding formulas such as SF and DSF and their impact on cardiometabolic parameters in patients with diabetes continues to generate interest and controversy, and there appears to be no consensus among researchers on the most effective management strategy for these patients.

DSFs usually contain carbohydrates with low GI such as fructose and large amounts of monounsaturated fatty acids in varying amounts, which have effect on glycaemic control [17-20]. On the other hand, SFs are often high in carbohydrate and contain only low to moderate levels of lipids and do not have dietetic fibre [17].

Previous reviews on the use of enteral nutrition in patients with diabetes $[16,17,21-23]$ either lacked consensus in the recommendations, were based only on glycaemic control or did not involve meta-analysis. In addition, concerns remain with the use of DSF in terms of the safety and tolerance of relatively high levels of fat and fructose with respect to lipid metabolism and lactic acidosis, despite its advantage in improving blood glucose compared with SFs $[16,19]$. Therefore, this review provides a quantitative assessment of the relative effectiveness of DSF compared with SF.

Aim: The aim was to evaluate the effectiveness of diabetes specific enteral nutrition formula versus SF in managing cardiometabolic parameters in patients with type 2 diabetes.

\section{Methods}

This study was conducted in accordance with the preferred reporting items for systematic reviews and meta-analyses (PRISMA) [24].

\subsection{Types of Studies and Participants}

Only randomised controlled studies were included in this review and participants were patients with type 2 diabetes.

Inclusion and Exclusion Criteria.

The criteria for considering studies for the review are outlined in Table 1.

Table 1. Criteria for considering studies for the review based on the Population, Intervention, Comparator, Outcomes and Study designs (PICOS) Structure.

\begin{tabular}{ccc}
\hline & Inclusion Criteria & Exclusion Criteria \\
\hline Population & $\begin{array}{c}\text { Patients with type 2 diabetes and on } \\
\text { enteral nutrition irrespective of type } \\
\text { of feeding tube. }\end{array}$ & $\begin{array}{c}\text { Patients with type 1 diabetes. } \\
\text { Healthy individuals without diabetes on enteral nutrition. } \\
\text { Patients with diabetes on parenteral nutrition and } \\
\text { parenteral plus enteral nutrition. } \\
\text { Studies involving animals }\end{array}$ \\
\hline Intervention & $\begin{array}{c}\text { Diabetes specific formulas } \\
\text { (Oral nutrition supplement or } \\
\text { enteral tube feeding) }\end{array}$ & Parenteral nutrition, parenteral plus enteral nutrition. \\
\hline Comparator & $\begin{array}{c}\text { Standard formulas (Oral nutrition } \\
\text { supplement or enteral tube feeding) }\end{array}$ & Parenteral nutrition and parenteral plus enteral nutrition. \\
\hline Outcomes & Cardiometabolic parameters & Qualitative outcomes such as patient feelings. \\
\hline Study Design: & Randomised Controlled Trials & Letters, comments, reviews, qualitative studies \\
\hline
\end{tabular}




\subsection{Type of Intervention}

The intervention for this review was based on diabetes-specific enteral formula, irrespective of the type of feeding tube, mode and rate of delivery of the enteral feed and clinical settings.

\subsection{Types of Outcome Measures}

The following were the outcome measures of interest;

- Blood glucose parameters-Fasting blood glucose and glycated haemoglobin.

- Lipid profile: Total cholesterol, low density lipoprotein (LDL) cholesterol, high density lipoprotein (HDL) cholesterol and triglycerides.

\subsection{Search Strategy}

Databases encompassing Pubmed, EMBASE, PSYCInfo and Google scholar were searched for relevant articles based on the Population (Patients with diabetes), Intervention (Diabetes Specific Formula), Comparator (Standard enteral formulas), Outcomes (outcome measures) and Study designs (Randomised controlled studies)-PICOS framework (Table 2) [25]. The use of key words, truncation symbols, Medical Subject Heading (MeSH) terms and Boolean operators (AND/OR) formed part of the search strategy. Searches were conducted from the date of inception of databases until 2 January 2019.

The screening of studies and the evaluation of their eligibility and inclusion were in line with PRISMA [24] guidelines (Figure 1). These procedures were conducted by five researchers (OO, SMW, $\mathrm{TT}, \mathrm{RC}, \mathrm{X}-\mathrm{HW}$ ) and differences were resolved through consensus.

Table 2. Search method for identification of studies.

\begin{tabular}{|c|c|c|c|c|}
\hline Patient/Population & Intervention & Comparator & Study Designs & $\begin{array}{l}\text { Combining } \\
\text { Search Terms }\end{array}$ \\
\hline $\begin{array}{l}\text { Patients with type } \\
2 \text { diabetes }\end{array}$ & $\begin{array}{l}\text { Diabetes specific } \\
\text { formulas }\end{array}$ & $\begin{array}{l}\text { Standard } \\
\text { formulas }\end{array}$ & $\begin{array}{l}\text { Randomised } \\
\text { Controlled Trial }\end{array}$ & \\
\hline $\begin{array}{l}\text { Type } 2 \text { diabetes OR } \\
\text { type } 2 \text { diabetes } \\
\text { mellitus OR } \\
\text { Diabetes } \\
\text { complications OR } \\
\text { diabetes mellitus, } \\
\text { type } 2\end{array}$ & $\begin{array}{l}\text { Diabetes specific } \\
\text { formula OR Diabetes } \\
\text { specific form* OR } \\
\text { Enteral nutrition OR } \\
\text { Enteral* OR Enteral }^{*} \text { feed OR Enteral feed* } \\
\text { OR Enteral form* OR } \\
\text { Diabetes formula OR } \\
\text { tube feeding OR } \\
\text { enteral feeding }\end{array}$ & & $\begin{array}{c}\text { Randomised } \\
\text { Controlled Trial OR } \\
\text { Randomized } \\
\text { Controlled Trial OR } \\
\text { Randomized } \\
\text { Controlled study OR } \\
\text { RCT OR Randomized* } \\
\text { OR controlled clinical } \\
\text { trial OR placebo OR } \\
\text { randomly OR trial OR } \\
\text { groups }\end{array}$ & $\begin{array}{c}\text { Column } 1 \text { AND } \\
\text { Column } 2 \text { AND } \\
\text { Column } 3\end{array}$ \\
\hline
\end{tabular}



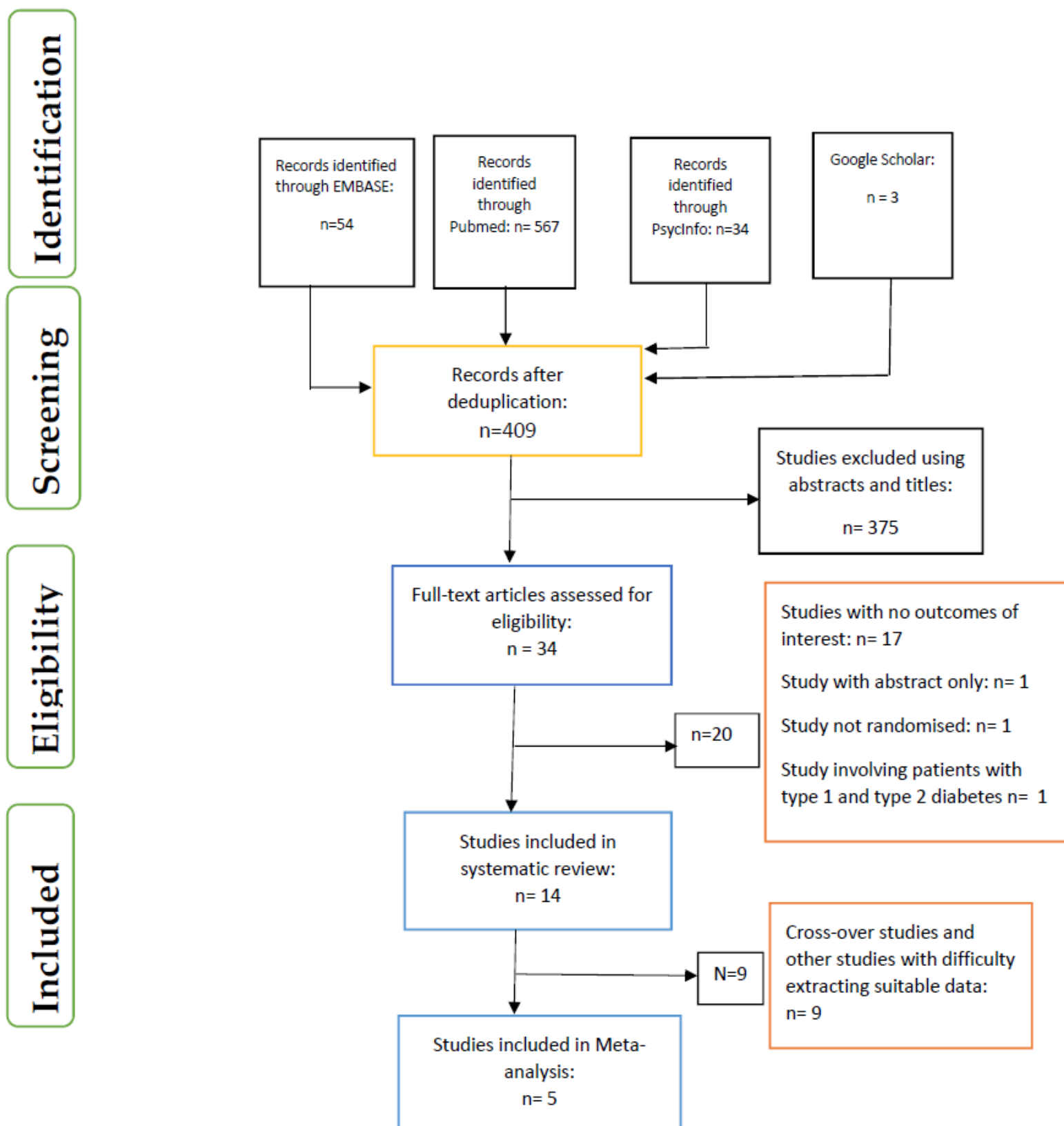

Figure 1. PRISMA flow chart on selection and inclusion of studies.

\subsection{Data Extraction}

All the articles from different databases were exported to ENDNote (Analytics, Philadelphia, PA, USA) for de-duplication. Data extraction was carried out by one researcher (OO) and cross-checked by the other four researchers (SMW, TT, RC, X-HW).

\subsection{Assessment of Risk of Bias and Evaluation of Quality}

A critical appraisal skills programme (CASP) tool was used to appraise the quality of the articles [26]. In addition, the researchers carried out an assessment of the risk of bias using the domain-based tool (random sequence generation, allocation concealment, blinding of participants, personnel and outcome assessment, reporting bias and selective reporting) to evaluate the studies included [27]. 


\subsection{Statistical Analysis}

Articles that met the inclusion criteria for meta-analysis were exported to RevMan (Review Manager, 5.3) [28] for data analysis. Therefore, cross-over studies and other studies which presented with difficulty in extracting suitable data were excluded from the meta-analysis. The data analysis included both meta-analysis and sensitivity analysis, the latter being conducted to test the consistency of the effect of DSFs on the different cardiometabolic paramters. The random effects model was used for the parameters of interest due to the high level of heterogeneity measured by the statistic $I^{2}$ with values ranging from $34 \%$ to $100 \%$. A p value of 0.10 was used to determine the statistical significance of heterogeneity.

\subsection{Effect Size}

A forest plot was used to present the results of the meta-analysis and statistical significance for the overall effect of the intervention was determined by a $p$ value of $<0.05$.

\section{Results}

\subsection{Data Inclusion Decisions}

Fasting blood glucose in the studies included was measured after overnight fasting, using standard measuring instruments. This is the standard method of measuring fasting blood glucose: the blood glucose concentrations were expressed as Means. However, the studies by Pohl et al. $[29,30]$ were expressed as median and interquartile ranges and these were converted to means and standard deviations [27]. Fourteen studies were included in the systematic review (Table 3) while only five studies [29-33] were selected for the meta-analysis (Table 4). 
Table 3. Characteristics of the articles included in this review $(n=14)$.

\begin{tabular}{|c|c|c|c|c|c|c|c|c|}
\hline Citation & Country & $\begin{array}{l}\text { Length of } \\
\text { Study }\end{array}$ & Study Type/Design & $\begin{array}{c}\text { Sample } \\
\text { Size/Description }\end{array}$ & Age (Years) & $\begin{array}{c}\text { Type of Enteral } \\
\text { Formula/Feeding Method }\end{array}$ & $\begin{array}{l}\text { Duration of } \\
\text { Diabetes } \\
\text { (Years) }\end{array}$ & Study Results/Conclusion \\
\hline $\begin{array}{l}\text { Ceriello et al. } \\
{[18]}\end{array}$ & Netherlands & $24 \mathrm{~h}$ & $\begin{array}{l}\text { Randomized, controlled, } \\
\text { double-blind, cross-over }\end{array}$ & $n=11$ & Mean \pm SEM & $\begin{array}{l}\text { The DSF had } 1 \mathrm{kcal} / \mathrm{mL} \text { and } \\
\text { low GI and/or slowly } \\
\text { digestible CHO. The SF was } \\
\text { isocaloric fibre containing } \\
\text { formula. } \\
\text { Bolus Feeding }\end{array}$ & Mean \pm SEM & $\begin{array}{l}\text { Administration of DSF } \\
\text { lowered glucose profiles. } \\
\text { Using DSF resulted in } \\
\text { significantly lower } 24 \mathrm{~h} \text { and } \\
\text { postprandial glucose profiles } \\
\text { than fibre-containing SF after } \\
\text { bolus administration. }\end{array}$ \\
\hline $\begin{array}{c}\text { Buranapin et al. } \\
{[20]}\end{array}$ & Thailand & $180 \mathrm{~min}$ & $\begin{array}{l}\text { Single centre, prospective, } \\
\text { randomized, double blind, } \\
\text { cross-over study. } \\
\text { Administration of oral DSF } \\
\text { and SF }\end{array}$ & $n=30$ & $\begin{array}{l}\text { Mean } \pm \text { SD } \\
60.93 \pm 11.71\end{array}$ & $\begin{array}{l}55 \% \text { CHO, } 15 \% \text { protein, } 30 \% \\
\text { fat for DSF and SF. However, } \\
\text { DSF substituted sucrose for } \\
\text { combination of fructose, } \\
\text { polydextrose and FOS. Bolus } \\
\text { Feeding }\end{array}$ & $\begin{array}{l}\text { More than } 6 \\
\text { months. }\end{array}$ & $\begin{array}{l}\text { DSF resulted in significantly } \\
\text { lower postprandial blood } \\
\text { glucose concentration than } \\
\text { SF. }\end{array}$ \\
\hline Pohl et al. [29] & Germany & 12 weeks & $\begin{array}{l}\text { Randomized, double-blind, } \\
\text { controlled, multi-centre trial. }\end{array}$ & $n=78$ & $\begin{array}{l}\text { Median (Range) } \\
\\
\text { Test group } \\
\text { (DSF): } 71 \text { (42-86) } \\
\text { Control group } \\
\text { (SF): } 72 \text { (51-87) }\end{array}$ & $\begin{array}{l}\text { DSF contained } 37 \% \text { energy as } \\
\text { CHO, } 45 \% \text { as fat, } 18 \% \text { as } \\
\text { protein, } \mathrm{SF} \text { contained } 52 \% \\
\text { energy as } \mathrm{CHO}, 30 \% \text { of } \\
\text { energy as total fat and } 18 \% \text { as } \\
\text { protein. } \\
\text { Continuous Feeding. }\end{array}$ & No data & $\begin{array}{l}\text { DSF formula resulted in a } \\
\text { more effective glycaemia } \\
\text { control than SF, and was } \\
\text { comparable in safety. } \\
\text { DSF significantly decreased } \\
\text { triglycerides compared with } \\
\text { SF, but differences were not } \\
\text { significant in relation to total } \\
\text { cholesterol, HDL and LDL } \\
\text { cholesterols. }\end{array}$ \\
\hline Pohl et al. [30] & Germany & 84 days & $\begin{array}{l}\text { Parallel design. } \\
\text { Stage two of a randomized, } \\
\text { prospective, double-blind, } \\
\text { controlled, multicentre, } \\
\text { parallel group study }\end{array}$ & $n=97$ & $\begin{array}{l}\text { DSF: } 74(44-91) \\
\text { SF: } 69 \text { (53-86) }\end{array}$ & $\begin{array}{l}\text { DSF contained } 37 \% \text { energy as } \\
\text { CHO, } 45 \% \text { as fat, } 18 \% \text { as } \\
\text { protein, } \mathrm{SF} \text { contained } 52 \% \\
\text { energy as CHO, } 30 \% \text { of } \\
\text { energy as total fat and } 18 \% \text { as } \\
\text { protein. }\end{array}$ & No data & $\begin{array}{c}\text { Compared to SF, DSF } \\
\text { significantly lowered FBG } \\
\text { and improved glycaemic } \\
\text { control. } \\
\text { There were no significant } \\
\text { differences between the two } \\
\text { groups with respect to TG, } \\
\text { TC, HDL and LDL } \\
\text { cholesterols. }\end{array}$ \\
\hline
\end{tabular}


Table 3. Cont.

\begin{tabular}{|c|c|c|c|c|c|c|c|c|}
\hline Citation & Country & $\begin{array}{l}\text { Length of } \\
\text { Study }\end{array}$ & Study Type/Design & $\begin{array}{c}\text { Sample } \\
\text { Size/Description }\end{array}$ & Age (Years) & $\begin{array}{c}\text { Type of Enteral } \\
\text { Formula/Feeding Method }\end{array}$ & $\begin{array}{l}\text { Duration of } \\
\text { Diabetes } \\
\text { (Years) }\end{array}$ & Study Results/Conclusion \\
\hline Craig et al. [31] & $\begin{array}{l}\text { USA-New York } \\
\text { State }\end{array}$ & 3 months & $\begin{array}{l}\text { Randomized, double-blind, } \\
\text { controlled, parallel group } 3 \\
\text { months pilot trial. }\end{array}$ & $n=34$ & $\begin{array}{c}80 \pm 2 \text { (range-SF: } \\
52-100)\end{array}$ & $\begin{array}{c}\text { Per } 1000 \mathrm{~mL} \text {, DSF contained } \\
1000 \mathrm{kcal}, 41.8 \mathrm{~g} \text { protein, } 93.7 \\
\mathrm{~g} \mathrm{CHO}, 55.7 \mathrm{~g} \text { fat. SF } \\
\text { contained } 1060 \mathrm{kcal}, 44.4 \mathrm{~g} \\
\text { protein, } 151.7 \mathrm{CHO}, 35.9 \mathrm{~g} \text { fat. } \\
\text { Continuous or intermittent } \\
\text { feeding. }\end{array}$ & No data & $\begin{array}{c}\text { DSF resulted in lower fasting } \\
\text { serum glucose and HbA1c } \\
\text { than SF. } \\
\text { No significant differences } \\
\text { between the DSF and SF } \\
\text { groups with respect to LDL } \\
\text { cholesterol and TG } 3 \text { months } \\
\text { post intervention, but the } \\
\text { DSF group had significantly } \\
\text { higher level of HDL } \\
\text { cholesterol than the SF group. }\end{array}$ \\
\hline \multirow[t]{2}{*}{$\begin{array}{l}\text { Lansink et al. } \\
\text { [32] }\end{array}$} & \multirow[t]{2}{*}{ Netherlands } & \multirow[t]{2}{*}{4 weeks } & \multirow[t]{2}{*}{$\begin{array}{l}\text { Randomized, controlled, } \\
\text { double-blind, parallel-group } \\
\text { study. }\end{array}$} & \multirow[t]{2}{*}{$n=44$} & Mean \pm SD & $\begin{array}{c}\text { DSF contained } 1 \mathrm{kcal} / \mathrm{mL}, 47 \\
\text { Energy } \% \text { CHO, } 19 \text { Energy } \% \\
\text { protein, } 34 \text { Energy } \% \text { fat and } 2 \\
\mathrm{~g} \text { fibres } / 100 \mathrm{~mL} \text {. The SF } \\
\text { contained } 50 \text { Energy } \% \text { CHO, } \\
16 \text { Energy } \% \text { protein, } 34 \\
\text { Energy } \% \text { fat and } 1.5 \mathrm{~g} \\
\text { fibres } / 100 \mathrm{~mL} .\end{array}$ & $\begin{array}{c}\text { Mean (Range) } \\
\text { DSF: } \\
84 \text { (18-216) } \\
\text { months }\end{array}$ & \multirow{2}{*}{$\begin{array}{l}\text { DSF significantly lowered } \\
\text { postprandial glucose } \\
\text { compared with SF. } \\
\\
\text { Levels of TG, TC, HDL and } \\
\text { LDL cholesterols were not } \\
\text { significantly different } \\
\text { between the two groups at } \\
\text { baseline and } 4 \text { weeks post } \\
\text { intervention. }\end{array}$} \\
\hline & & & & & $\begin{array}{l}\text { DSF: } 65.2 \pm 7.4 \\
\text { SF: } 64.2 \pm 5.9\end{array}$ & Bolus Feeding & $\begin{array}{l}\text { SF: } 66(10-504) \\
\text { months }\end{array}$ & \\
\hline \multirow{3}{*}{$\begin{array}{l}\text { Vaisman et al. } \\
\text { [33] }\end{array}$} & \multirow{3}{*}{ No data } & \multirow{3}{*}{12 weeks } & \multirow{3}{*}{$\begin{array}{c}\text { Randomized, controlled, } \\
\text { double-blind, parallel group } \\
\text { study. }\end{array}$} & $n=25$ & $\begin{array}{l}\text { Total: } 76.2 \pm \\
12.8 \text { years }\end{array}$ & $\begin{array}{l}\text { DSF contained } 100 \mathrm{kcal}, 45 \\
\text { Energy } \% \text { CHO, } 38 \text { Energy } \% \\
\text { fat, } 17 \mathrm{Energy} \% \text { protein and } \\
1.5 \mathrm{~g} / 100 \mathrm{kcal} \text { fibre. SF } \\
\text { contained } 100 \mathrm{kcal}, 55 \\
\text { Energy } \% \text { CHO, } 30 \text { Energy } \% \\
\text { fat, } 15 \text { Energy } \% \text { protein, } 2 \\
\text { g/100 kcal fibre. }\end{array}$ & Mean \pm SD & \multirow{3}{*}{$\begin{array}{l}\text { The DSF significantly } \\
\text { reduced HbA1c compared to } \\
\text { SF. No significant effect was } \\
\text { found with respect to fasting } \\
\text { blood glucose. } \\
\\
\text { DSF significantly increased } \\
\text { HDL cholesterol, but } \\
\text { differences were not } \\
\text { significant in relation to TG, } \\
\text { TC and LDL cholesterol } \\
\text { compared with SF. }\end{array}$} \\
\hline & & & & & DSF: $73.0 \pm 14.7$ & $\begin{array}{l}\text { Bolus, Continuous or } \\
\text { intermittent feeding. }\end{array}$ & $\begin{array}{l}\text { Total: } 8.6 \pm 7.6 \\
\quad \text { years } \\
\text { DSF: } 5.0 \pm 4.9\end{array}$ & \\
\hline & & & & & SF: $79.2 \pm 10.4$ & \multicolumn{2}{|c|}{ SF: $12.6 \pm 8.4$} & \\
\hline
\end{tabular}


Table 3. Cont.

\begin{tabular}{|c|c|c|c|c|c|c|c|c|}
\hline Citation & Country & $\begin{array}{l}\text { Length of } \\
\text { Study }\end{array}$ & Study Type/Design & $\begin{array}{c}\text { Sample } \\
\text { Size/Description }\end{array}$ & Age (Years) & $\begin{array}{c}\text { Type of Enteral } \\
\text { Formula/Feeding Method }\end{array}$ & $\begin{array}{c}\text { Duration of } \\
\text { Diabetes } \\
\text { (Years) }\end{array}$ & Study Results/Conclusion \\
\hline Alish et al. [34] & USA & 10 days & $\begin{array}{l}\text { Randomized, double blind, } \\
\text { two treatment, crossover } \\
\text { design. } \\
\text { DSF (Postprandial response } \\
\text { protocol) vs. SF (Continuous } \\
\text { glucose monitoring). }\end{array}$ & $n=12$ & $\begin{array}{l}\text { Mean } \pm \text { SEM } \\
\\
\text { Postprandial: } \\
63.1 \pm 1.9 \\
\text { Continuous } \\
\text { feed: } 74.1 \pm 4.0\end{array}$ & $\begin{array}{c}\text { DSF had } 1.2 \mathrm{kcal} / \mathrm{mL}, 114.5 \mathrm{~g} \\
\text { CHO, } 17 \mathrm{~g} / \mathrm{L} \text { fibre, } 60 \mathrm{~g} / \mathrm{L} \\
\text { protein, } 60 \mathrm{~g} / \mathrm{L} \text { fat. SF had } 1.2 \\
\mathrm{kcal} / \mathrm{mL}, 169.4 \mathrm{~g} \text { CHO, } 18 \mathrm{~g} / \mathrm{L} \\
\text { fibre, } 55.5 \mathrm{~g} / \mathrm{L} \text { protein, } 39.3 \\
\mathrm{~g} / \mathrm{L} \text { fat. } \\
\text { Continuous Feeding. }\end{array}$ & NS & $\begin{array}{l}\text { Use of DSF produced lower } \\
\text { postprandial glycaemic and } \\
\text { insulinemic responses, } \\
\text { reduced glycaemic } \\
\text { variability, and resulted in } \\
\text { less hyperglycaemia, reduced } \\
\text { short acting insulin } \\
\text { requirements. }\end{array}$ \\
\hline Gulati et al. [35] & India & 8 months & $\begin{array}{l}\text { Open-label, randomized, } \\
\text { crossover, pilot single centre } \\
\text { study. }\end{array}$ & $n=40$ & $35-60$ years & $\begin{array}{l}\text { DSF administered was } 55 \mathrm{~g} \text { in } \\
210 \mathrm{~mL} \text { of water to make } 250 \\
\mathrm{~mL} \text { at standard reconstitution } \\
\text { ( } 1 \mathrm{kcal} / \mathrm{mL}) \text { which can be } \\
\text { used as tube feed or oral } \\
\text { nutrition supplement. The SF } \\
\text { was isocaloric Meal. }\end{array}$ & No data & $\begin{array}{l}\text { The level of HDL cholesterol } \\
\text { was significantly higher in } \\
\text { the DSF group compared } \\
\text { with the SF group after } \\
\text { intervention, but differences } \\
\text { were not significant in } \\
\text { relation to TG, TC and LDL } \\
\text { cholesterol. }\end{array}$ \\
\hline $\begin{array}{l}\text { Hofman et al. } \\
{[36]}\end{array}$ & Netherlands & $360 \mathrm{~min}$ & $\begin{array}{l}\text { Randomized, double blind, } \\
\text { cross over study involving SF } \\
\text { (A), DSF with moderate } \\
\text { amount of carbohydrate and } \\
\text { MUFA (B) and Test feed with } \\
\text { low amount of carbohydrate } \\
\text { and high amount of fat (C) }\end{array}$ & $n=12$ & $63 \pm 9.4$ years & $\begin{array}{l}\text { DSF (45 Energy\% CHO, } 26 \\
\text { Energy\% MUFA), SF (49 } \\
\text { Energy\% CHO, } 21 \text { Energy\% } \\
\text { MUFA). Continuous Feeding. }\end{array}$ & No data & $\begin{array}{l}\text { DSF showed significantly } \\
\text { lower glucose levels } \\
\text { compared with SF. }\end{array}$ \\
\hline
\end{tabular}


Table 3. Cont.

\begin{tabular}{|c|c|c|c|c|c|c|c|c|}
\hline Citation & Country & $\begin{array}{l}\text { Length of } \\
\text { Study }\end{array}$ & Study Type/Design & $\begin{array}{c}\text { Sample } \\
\text { Size/Description }\end{array}$ & Age (Years) & $\begin{array}{c}\text { Type of Enteral } \\
\text { Formula/Feeding Method }\end{array}$ & $\begin{array}{l}\text { Duration of } \\
\text { Diabetes } \\
\text { (Years) }\end{array}$ & Study Results/Conclusion \\
\hline \multirow[t]{2}{*}{$\begin{array}{c}\text { Lansink et al. } \\
{[37]}\end{array}$} & \multirow[t]{2}{*}{ Netherlands } & \multirow[t]{2}{*}{$8 \mathrm{~h}$} & \multirow[t]{2}{*}{$\begin{array}{c}\text { Randomized, controlled, } \\
\text { double-blind cross-over } \\
\text { study }\end{array}$} & \multirow[t]{2}{*}{$n=24$} & Mean \pm SD & $\begin{array}{l}\text { The DSF had } 1.5 \mathrm{kcal} / \mathrm{mL} \text {, } \\
\text { high protein, a mixture of } 6 \\
\text { different dietary fibre and } \\
\text { low GI CHO. SF was } \\
\text { isocaloric fibre containing } \\
\text { formula. }\end{array}$ & $\begin{array}{l}\text { Median } \\
\text { (Minimum and } \\
\text { Maximum) }\end{array}$ & \multirow{2}{*}{$\begin{array}{l}\text { Administration of a new, } \\
\text { high-protein DSF during } 4 \mathrm{~h} \\
\text { of continuous feeding } \\
\text { resulted in lower glucose and } \\
\text { insulin levels compared with } \\
\text { a fiber-containing SF. DSF } \\
\text { may contribute to lower } \\
\text { glucose levels in these } \\
\text { patients. }\end{array}$} \\
\hline & & & & & $64.6 \pm 10.7$ & Continuous Feeding. & $\begin{array}{l}76.5 \text { months }(13, \\
\text { 303) }\end{array}$ & \\
\hline \multirow{2}{*}{$\begin{array}{l}\text { Mesejo et al. } \\
\text { [38] }\end{array}$} & \multirow[t]{2}{*}{ Spain } & \multirow[t]{2}{*}{2 years } & \multirow{2}{*}{$\begin{array}{l}\text { Prospective, open-label, } \\
\text { randomized study }\end{array}$} & \multirow[t]{2}{*}{$n=157$} & $\begin{array}{l}\text { Median } \\
\text { (Q1-Q3) }\end{array}$ & $\begin{array}{l}\text { Per } 100 \mathrm{~mL}, \mathrm{DSF} \text { had } 100 \\
\text { kcal, } 5.7 \mathrm{~g} \text { protein, } 8.2 \mathrm{~g} \mathrm{CHO} \text {, } \\
4.4 \mathrm{~g} \text { fat. SF had } 100 \mathrm{kcal}, 5 / 7 \\
\mathrm{~g} \text { protein, } 10.93 / 15.3 \mathrm{~g} \mathrm{CHO}, \\
3.79 / 5.3 \mathrm{~g} \text { fat. }\end{array}$ & \multirow{4}{*}{ No data } & $\begin{array}{l}\text { DSFs lowered insulin } \\
\text { requirements, improved } \\
\text { glycaemic control and } \\
\text { reduced the risk of acquired } \\
\text { infections relative to SF. }\end{array}$ \\
\hline & & & & & $\begin{array}{l}\text { New generation } \\
\text { DSF: } 57(43-70) \\
\text { SF: } 60 \text { (45-71) } \\
\text { Control DSF: } 58 \\
\quad(46-68)\end{array}$ & Continuous Feeding. & & $\begin{array}{l}\text { Plasma levels of cholesterol } \\
\text { and TG were similar across } \\
\text { the three treatment groups. }\end{array}$ \\
\hline \multirow[t]{2}{*}{ Voss et al. [39] } & \multirow[t]{2}{*}{ USA } & \multirow[t]{2}{*}{$240 \mathrm{~min}$} & $\begin{array}{l}\text { Randomized cross } \\
\text { over-study }\end{array}$ & \multirow[t]{2}{*}{$n=48$} & Mean \pm SEM & $\begin{array}{c}\text { DSF had } 1 \mathrm{kcal} / \mathrm{mL}, 47.8 \mathrm{~g} \\
\text { CHO, } 7.2 \mathrm{~g} \text { fibre, } 20.9 \mathrm{~g} \\
\text { protein, } 27.2 \mathrm{~g} \text { fat. SF had } \\
1.06 \mathrm{kcal} / \mathrm{mL}, 73 \mathrm{~g} \mathrm{CHO}, 7.2 \mathrm{~g} \\
\text { fibre, } 20.9 \mathrm{~g} \text { protein, } 16.4 \mathrm{~g} \text { fat. }\end{array}$ & & \multirow[t]{2}{*}{$\begin{array}{l}\text { DSF resulted in lower } \\
\text { postprandial blood glucose } \\
\text { response compared with SF. }\end{array}$} \\
\hline & & & $\begin{array}{l}\text { Double-blinded with } \\
\text { three-treatments }\end{array}$ & & $56 \pm 1.4$ years & Bolus Feeding & & \\
\hline \multirow[t]{2}{*}{$\begin{array}{l}\text { Vanschoonbeek } \\
\text { et al. [40] }\end{array}$} & \multirow[t]{2}{*}{ Netherlands } & \multirow[t]{2}{*}{10 days } & \multirow[t]{2}{*}{$\begin{array}{l}\text { Randomized, double-blind, } \\
\text { cross over study. }\end{array}$} & \multirow[t]{2}{*}{$n=15$} & Mean \pm SEM & $\begin{array}{c}\text { Per } 100 \mathrm{~mL} \text {, DSF had } 98 \mathrm{kcal} \text {, } \\
1.44 \mathrm{~g} \text { fibre and } 5.44 / 50 \\
\text { (g/energy\%) of fat. SF had } \\
100 \mathrm{kcal}, 1.4 \mathrm{~g} \text { of fibre, } 3.4 / 30 \\
\text { (g/energy } \% \text { ) of fat.), }\end{array}$ & Mean \pm SEM & \multirow[t]{2}{*}{$\begin{array}{l}\text { DSF rich in lowly digestible } \\
\text { carbohydrate sources can be } \\
\text { equally effective in lowering } \\
\text { the postprandial blood } \\
\text { glucose response as } \\
\text { low-carbohydrate, high-fat } \\
\text { enteral formulas without } \\
\text { elevating the plasma } \\
\text { triglyceride response. }\end{array}$} \\
\hline & & & & & $63 \pm 1$ years & Bolus Feeding & $9 \pm 2$ years & \\
\hline
\end{tabular}

Abbreviations: NS (Not stated); DSF (Diabetes Specific Formula); CHO (Carbohydrate); FOS (Fructo-oligosaccharide); GI (Glycaemic Index); HbA1c (Glycated haemoglobin); SF (Standard Formula); LDL (low density lipoprotein) Cholesterol; HDL (high density lipoprotein)Cholesterol; MUFA (mono-unsaturated fatty acid); FBG (fasting blood glucose); TC (total cholesterol); TG (triglycerides); T2DM (type 2 diabetes mellitus). 
Table 4. Blood glucose parameters among individuals with diabetes (Meta-analysis Data Extraction Table).

\begin{tabular}{|c|c|c|c|c|c|c|c|c|}
\hline $\begin{array}{c}\text { Study } \\
\text { Reference }\end{array}$ & Interventions & $\begin{array}{l}\text { Pre-and Post } \\
\text { Intervention }\end{array}$ & $\begin{array}{l}\text { Fasting Blood } \\
\text { Glucose } \\
\text { mmol/L Mean } \\
\pm \text { SD/Median } \\
\text { (Quartiles) }\end{array}$ & $\begin{array}{c}\text { Glycated } \\
\text { Haemoglobin } \\
\% \text { Mean } \pm \\
\text { SD/Median } \\
\text { (Quartiles) }\end{array}$ & $\begin{array}{c}\text { Total } \\
\text { Cholesterol } \\
\text { mmol/L Mean } \\
\pm \text { SD/Median } \\
\text { (Quartiles) }\end{array}$ & $\begin{array}{c}\text { LDL } \\
\text { Cholesterol } \\
\text { mmol/L Mean } \\
\pm \text { SD/Median } \\
\text { (Quartiles) }\end{array}$ & $\begin{array}{c}\text { HDL } \\
\text { Cholesterol } \\
\text { mmol/L Mean } \\
\pm \text { SD/Median } \\
\text { (Quartiles) }\end{array}$ & $\begin{array}{c}\text { Triglycerides } \\
\text { mmol/L Mean } \\
\pm \text { SD/Median } \\
\text { (Quartiles) }\end{array}$ \\
\hline \multirow[t]{2}{*}{ Pohl. et al. [29] } & DSF, $n=39$ & $\begin{array}{c}\text { Change from } \\
\text { baseline }\end{array}$ & $\begin{array}{c}* * \Delta-1.59(-3.38 \\
\text { to }-0.06)\end{array}$ & $\begin{array}{c}* * \Delta-0.8(-1.5 \text { to } \\
-0.5)\end{array}$ & $\begin{array}{c}* * \Delta-0.37(-1.00 \\
\text { to } 0.56)\end{array}$ & $\begin{array}{c}* * \Delta-0.28(-1.46 \\
\text { to } 0.53)\end{array}$ & $\begin{array}{c}* * \Delta 0.08(-0.06 \\
\text { to } 0.28)\end{array}$ & $\begin{array}{c}* *-0.37(-0.36 \\
\text { to } 0.38)\end{array}$ \\
\hline & $\mathrm{SF}, n=39$ & $\begin{array}{l}\text { Change from } \\
\text { baseline }\end{array}$ & $\begin{array}{c}* * \Delta-0.08(-1.34 \\
\text { to } 0.79)\end{array}$ & $\begin{array}{c}* * 0.0(-0.4 \text { to } \\
0.3)\end{array}$ & $\begin{array}{c}* * \Delta-0.23(-1.22 \\
\text { to } 0.46)\end{array}$ & $\begin{array}{c}* *-0.52(-1.48 \\
\text { to } 0.04)\end{array}$ & $\begin{array}{c}* * 0.05(-0.10 \\
\text { to } 0.32)\end{array}$ & $\begin{array}{c}* * 0.203(-0.07 \\
\text { to } 0.84)\end{array}$ \\
\hline \multirow[t]{2}{*}{ Pohl et al. [30] } & DSF, $n=48$ & $\begin{array}{l}\text { Change from } \\
\text { baseline }\end{array}$ & $\begin{array}{c}* * \Delta-2.17 \\
(-2.55 /-1.33)\end{array}$ & $\begin{array}{c}* * \Delta-1.30 \\
(-2.60 /-0.10)\end{array}$ & $\begin{array}{c}* * \Delta 0.30 \\
(-1.22 / 1.06)\end{array}$ & $\begin{array}{l}{ }^{* *} \Delta 0.27 \\
(-0.71 / 1.40\end{array}$ & $\begin{array}{c}{ }^{* *} \Delta 0.03 \\
(-0.26 / 0.4)\end{array}$ & $\begin{array}{c}* * \Delta-0.45 \\
(-1.65 / 0.27)\end{array}$ \\
\hline & $\mathrm{SF}, n=49$ & $\begin{array}{l}\text { Change from } \\
\text { baseline }\end{array}$ & $\begin{array}{c}* * \Delta-0.67 \\
(-0.90 /-0.10)\end{array}$ & $\begin{array}{c}* * \Delta-1.20 \\
(-2.35 /-0.55)\end{array}$ & $\begin{array}{l}* * \Delta 0.21 \\
(-1.02 / 0.48)\end{array}$ & $\begin{array}{l}* * \Delta-0.33 \\
(-1.03 / 0.56)\end{array}$ & $\begin{array}{c}* * \Delta 0.00 \\
(-0.22 / 0.28)\end{array}$ & $\begin{array}{l}* * \Delta-0.70 \\
(-1.50 / 1.73)\end{array}$ \\
\hline \multirow{4}{*}{ Craig et al. [31] } & & Baseline & $* 7.3 \pm 0.4$ & $* 6.9 \pm 0.3$ & $* 4.16 \pm 0.31$ & $* 2.66 \pm 0.23$ & $* 1.01 \pm 0.05$ & $* 0.97 \pm 0.13$ \\
\hline & & Final & $6.7 \pm 0.7$ & $6.5 \pm 0.4$ & $3.96 \pm 0.31$ & $2.51 \pm 0.28$ & $0.98 \pm 0.05$ & $0.91 \pm 0.17 \mathrm{~g} / \mathrm{L}$ \\
\hline & SF & Baseline & $* 6.9 \pm 0.6$ & $* 6.9 \pm 0.5$ & $* 4.21 \pm 0.18$ & $* 2.69 \pm 0.15$ & $* 0.98 \pm 0.05$ & $* 0.9 \pm 0.07$ \\
\hline & $S F, n=13$ & Final & $8.3 \pm 1.7$ & $6.9 \pm 0.14$ & $3.96 \pm 0.23$ & $2.53 \pm 0.21$ & $0.83 \pm 0.05$ & $1.06 \pm 0.12 \mathrm{~g} / \mathrm{L}$ \\
\hline \multirow{2}{*}{$\begin{array}{c}\text { Lansink et al. } \\
\text { [32] }\end{array}$} & $\mathrm{DSF}, n=21$ & $\begin{array}{l}\text { Baseline } \\
\text { Final }\end{array}$ & $\begin{array}{c}* 8.32 \pm 0.33 \\
8.13 \pm 0.33\end{array}$ & No data & No data & No data & No data & No data \\
\hline & $\mathrm{SF}, n=22$ & $\begin{array}{l}\text { Baseline } \\
\text { Final }\end{array}$ & $\begin{array}{c}* 7.73 \pm 0.22 \\
8.22 \pm 0.26\end{array}$ & No data & No data & No data & No data & No data \\
\hline \multirow{2}{*}{$\begin{array}{c}\text { Vaisman et al. } \\
{[33]}\end{array}$} & DSF, $n=12$ & $\begin{array}{l}\text { Baseline } \\
\text { Final }\end{array}$ & No data & $\begin{array}{c}* * * 6.9 \pm 0.3 \\
6.2 \pm 0.4\end{array}$ & No data & No data & $\begin{array}{c}* * * 1.04 \pm 0.08 \\
1.23 \pm 0.10\end{array}$ & No data \\
\hline & $\mathrm{SF}, n=13$ & $\begin{array}{l}\text { Baseline } \\
\text { Final }\end{array}$ & No data & $\begin{array}{c}* * * 7.9 \pm 0.3 \\
8.7 \pm 0.4\end{array}$ & No data & No data & $\begin{array}{c}* * * 1.06 \pm 0.08 \\
0.94 \pm 0.09\end{array}$ & No data \\
\hline
\end{tabular}

Abbreviations: NS (Not stated); SD (Standard deviation); SEM (Standard Error of Mean); $\Delta$ (Change from baseline) ${ }^{*}$ Mean \pm SD; ${ }^{* *}$ Median (Quartiles); ${ }^{* * *}$ Mean \pm SEM. 


\subsection{Assessment of Risk of Bias in Included Studies}

Figure 2 shows the risk of bias summary of the various studies included in the meta-analysis. All the studies demonstrated low risk of bias in all the areas, except with respect to incomplete outcome data (attrition bias) where two studies $[29,33]$ showed high risk of bias.

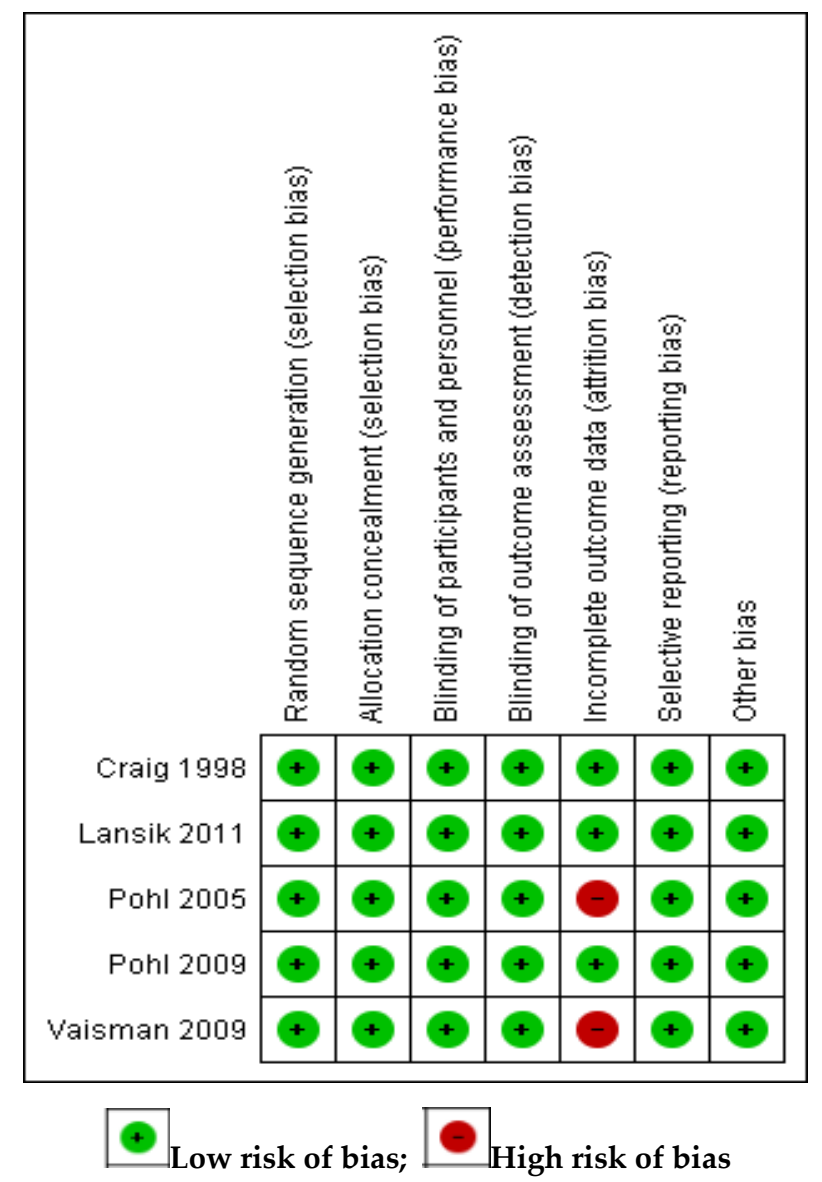

Figure 2. Risk of bias summary.

Based on the findings of the review and the meta-analysis, two distinct areas were evident: the effect of DSF on blood glucose parameters and the effect of DSF on lipid profile.

The effect DSF on blood glucose parameters:

All the fourteen studies included in the systematic review showed that DSF was effective in lowering blood glucose parameters in patients with type 2 diabetes compared with SF. In particular, DSF improved glycaemic control and lowered insulin requirements [18,29,31,35-39]. It provided better clinical outcomes, including reducing the risk of acquired infections and pressure ulcer, reduced body weight and was safer compared to SF $[29,31,32]$. In addition, the use of DSF was shown to be effective in lowering postprandial blood glucose levels compared to SF [20,32,34,40].

Pohl et al. [30] observed that long-term tube feeding with a DSF significantly lowered fasting blood glucose and improved glycaemic control. Similarly, Vaisman et al. [33] reported that DSF significantly improved longer-term glycaemic control in diabetic patients compared to SF. The results of the meta-analysis confirmed the findings of the systematic review. With respect to the fasting blood glucose, it was significantly lower $(p=0.01)$ in the DSF group compared to SF, with a mean difference of $-1.15(95 \% \mathrm{CI}-2.07,-0.23)$ (Figure 3$)$. However, in relation to the sensitivity analysis, there were no significant differences $(p>0.05)$ between the two groups with the removal of Pohl et al. $[29,30]$ studies. 


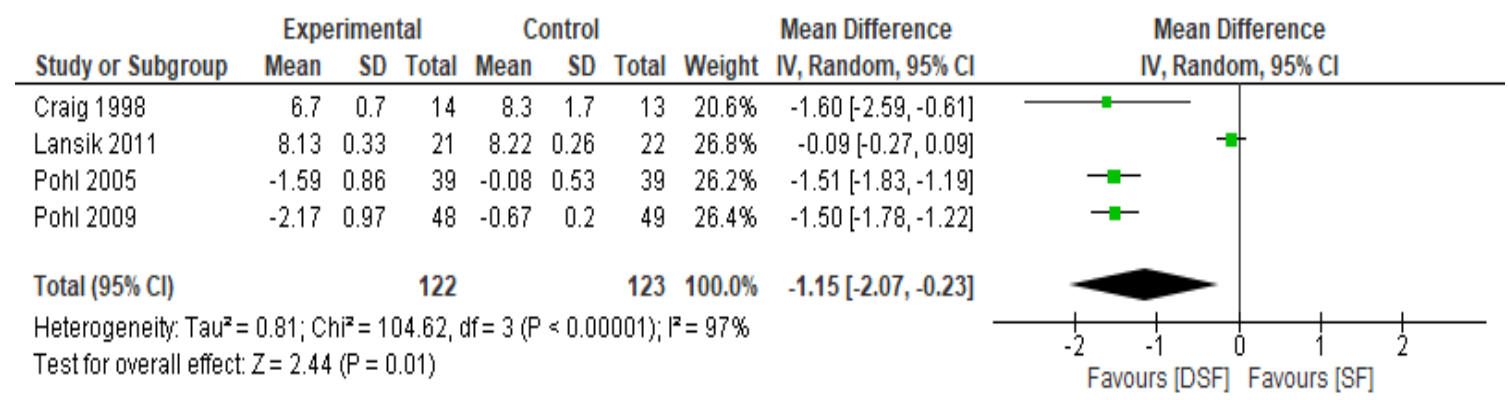

Figure 3. The effect of DSF on fasting blood glucose (mmol/L).

The glycated haemoglobin was significantly lower $(p=0.005)$ in the DSF group compared to the SF group following meta-analysis (Figure 4 ) and sensitivity analysis.

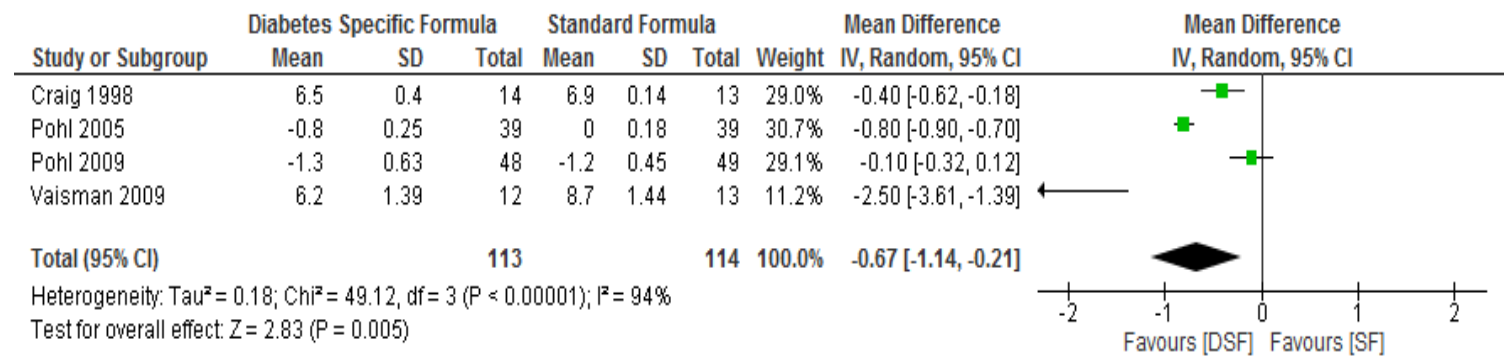

Figure 4. The effect of DSF on Glycated Haemoglobin \%.

\subsection{The Effect of DSF on Lipid Profile}

Based on the systematic review, the outcomes of the studies selected to evaluate the effect of DSF on lipid profile were variable. Craig et al. [31] did not find significant differences with respect to LDL cholesterol and triglyceride between the DSF and the SF groups, but differences were significantly higher $(p<0.05)$ in the DSF group in relation to HDL cholesterol. In two other studies $[33,35]$, the level of HDL cholesterol was significantly higher $(p<0.05)$ in the DSF group compared with the SF group after intervention, but differences were not significant $(p>0.05)$ in relation to triglycerides, total cholesterol and LDL cholesterols. Differences between DSF and SF were also not significant $(p>0.05)$ in terms of triglyceride, total cholesterol, HDL cholesterol and LDL cholesterol in other studies [30,32,38]. In contrast, Pohl et al. [29] reported that there was a significant difference $(p<0.05)$ between the DSF group and the SF group with respect to triglycerides, but differences were not significant $(p>0.05)$ in relation to total cholesterol, HDL and LDL cholesterol. Other studies $[36,40]$ have also shown that DSF is effective in controlling plasma triglyceride.

Following meta-analysis, no significant differences $(p>0.05)$ were found between the DSF and SF groups with respect to total cholesterol, LDL cholesterol and triglyceride (Figures 5-7). However, the DSF group had a significantly higher level $(p=0.04)$ of HDL cholesterol compared to the SF group after the intervention, with a mean difference of 0.09 (95\% CI, 0.00, 0.18) (Figure 8). The results of the sensitivity test for HDL cholesterol demonstrated no significant differences $(p>0.05)$ between the two groups when the Craig et al. [31] and Vaisman et al. [33] studies were removed from the analysis. In addition, the sensitivity analysis showed no significant differences $(p>0.05)$ between the two groups with respect to total cholesterol and triglyceride, while significant differences $(<0.05)$ were observed in relation to LDL cholesterol when the Craig et al. [31] study was removed. 


\begin{tabular}{|c|c|c|c|c|c|c|c|c|c|}
\hline \multirow[b]{2}{*}{ Study or Subgroup } & \multicolumn{3}{|c|}{ Diabetes Specific Formula } & \multicolumn{3}{|c|}{ Standard Formula } & & \multirow{2}{*}{$\begin{array}{l}\text { Mean Difference } \\
\text { IV, Random, } 95 \% \mathrm{Cl}\end{array}$} & \multirow{2}{*}{$\begin{array}{c}\text { Mean Difference } \\
\text { IV, Random, } 95 \% \mathrm{Cl}\end{array}$} \\
\hline & Mean & SD & Total & Mean & SD & Total & Weight & & \\
\hline Craig 1998 & 3.96 & 0.31 & 14 & 3.96 & 0.23 & 13 & $30.4 \%$ & $0.00[-0.20,0.20]$ & \\
\hline Pohl 2005 & -0.37 & 0.39 & 39 & -0.23 & 0.42 & 39 & $36.1 \%$ & $-0.14[-0.32,0.04]$ & \\
\hline Pohl 2009 & 0.3 & 0.57 & 48 & 0.21 & 0.36 & 49 & $33.6 \%$ & $0.09[-0.10,0.28]$ & \\
\hline Total $(95 \% \mathrm{Cl})$ & & & 101 & & & 101 & $100.0 \%$ & $-0.02[-0.16,0.12]$ & \\
\hline \multicolumn{10}{|c|}{ 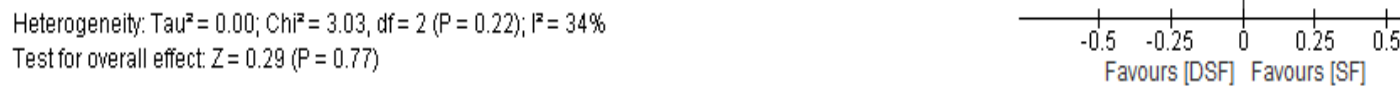 } \\
\hline
\end{tabular}

Figure 5. The effect of DSF on total cholesterol (mmol/L).

\begin{tabular}{|c|c|c|c|c|c|c|c|c|c|c|c|}
\hline \multirow[b]{2}{*}{ Study or Subgroup } & \multicolumn{3}{|c|}{ Diabetes Specific Formula } & \multicolumn{3}{|c|}{ Standard Formula } & \multirow[b]{2}{*}{ Weight } & \multirow{2}{*}{$\begin{array}{l}\text { Mean Difference } \\
\text { IV, Random, } 95 \% \mathrm{Cl}\end{array}$} & & \multirow{2}{*}{$\begin{array}{c}\text { Mean Difference } \\
\text { IV, Random, } 95 \% \mathrm{Cl}\end{array}$} & \\
\hline & Mean & SD & Total & Mean & SD & Total & & & & & \\
\hline Craig 1998 & 2.51 & 0.28 & 14 & 2.53 & 0.21 & 13 & $33.5 \%$ & $-0.02[-0.21,0.17]$ & & -4 & \\
\hline Pohl 2005 & -0.28 & 0.5 & 39 & -0.52 & 0.38 & 39 & $33.1 \%$ & $0.24[0.04,0.44]$ & & & \\
\hline Pohl 2009 & 0.27 & 0.53 & 48 & -0.33 & 0.4 & 49 & $33.4 \%$ & $0.60[0.41,0.79]$ & & - & \\
\hline Total $(95 \% \mathrm{Cl})$ & & & 101 & & & 101 & $100.0 \%$ & $0.27[-0.09,0.63]$ & & & \\
\hline \multicolumn{11}{|c|}{ 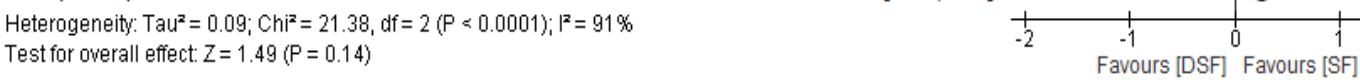 } & $\frac{1}{2}$ \\
\hline
\end{tabular}

Figure 6. The effect of DSF on LDL cholesterol (mmol/L).

\begin{tabular}{|c|c|c|c|c|c|c|c|c|c|}
\hline \multirow[b]{2}{*}{ Study or Subgroup } & \multicolumn{3}{|c|}{ Diabetes Specific Formula } & \multicolumn{3}{|c|}{ Standard Formula } & \multirow[b]{2}{*}{ Weight } & \multirow{2}{*}{$\begin{array}{l}\text { Mean Difference } \\
\text { IV, Random, } 95 \% \mathrm{Cl}\end{array}$} & \multirow{2}{*}{$\begin{array}{c}\text { Mean Difference } \\
\text { IV, Random, } 95 \% \mathrm{Cl}\end{array}$} \\
\hline & Mean & SD & Total & Mean & SD & Total & & & \\
\hline Craig 1998 & 1.03 & 0.19 & 14 & 1.12 & 0.14 & 13 & $34.2 \%$ & $-0.09[-0.22,0.04]$ & $\rightarrow+$ \\
\hline Pohl 2005 & -0.37 & 0.19 & 39 & 0.2 & 0.23 & 39 & $34.6 \%$ & $-0.57[-0.66,-0.48]$ & - \\
\hline Pohl 2009 & -0.45 & 0.48 & 48 & -0.7 & 0.81 & 49 & $31.2 \%$ & $0.25[-0.01,0.51]$ & $\longrightarrow$ \\
\hline Total $(95 \% \mathrm{Cl})$ & & & 101 & & & 101 & $100.0 \%$ & $-0.15[-0.59,0.29]$ & \\
\hline \multicolumn{10}{|c|}{$\begin{array}{l}\text { Heterogeneity: } \text { Tau }^{2}=0.14 ; C h i^{2}=57.54, d f=2(P<0.00001) ; l^{2}=97 \% \\
\text { Test for overall effect: } Z=0.67(P=0.50)\end{array}$} \\
\hline
\end{tabular}

Figure 7. The effect of DSF on Triglycerides (mmol/L).

\begin{tabular}{|c|c|c|c|c|c|c|c|c|c|c|}
\hline \multirow[b]{2}{*}{ Study or Subgroup } & \multicolumn{3}{|c|}{ Diabetes Specific Formula } & \multicolumn{3}{|c|}{ Standard Formula } & \multirow[b]{2}{*}{ Weight } & \multirow{2}{*}{$\begin{array}{l}\text { Mean Difference } \\
\text { IV, Random, } 95 \% \mathrm{Cl}\end{array}$} & \multirow{2}{*}{\multicolumn{2}{|c|}{$\begin{array}{c}\text { Mean Difference } \\
\text { IV, Random, } 95 \% \mathrm{Cl}\end{array}$}} \\
\hline & Mean & SD & Total & Mean & SD & Total & & & & \\
\hline Craig 1998 & 0.98 & 0.05 & 14 & 0.83 & 0.05 & 13 & $33.4 \%$ & $0.15[0.11,0.19]$ & & $\rightarrow$ \\
\hline Pohl 2005 & 0.08 & 0.09 & 39 & 0.05 & 0.11 & 39 & $32.7 \%$ & $0.03[-0.01,0.07]$ & & - \\
\hline Pohl 2009 & 0.03 & 0.17 & 48 & 0 & 0.3 & 49 & $25.2 \%$ & $0.03[-0.07,0.13]$ & & 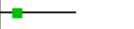 \\
\hline Vaisman 2009 & 1.23 & 0.35 & 12 & 0.94 & 0.32 & 13 & $8.8 \%$ & $0.29[0.03,0.55]$ & & \\
\hline Total $(95 \% \mathrm{Cl})$ & & & 113 & & & 114 & $100.0 \%$ & $0.09[0.00,0.18]$ & & \\
\hline \multicolumn{9}{|c|}{$\begin{array}{l}\text { Heterogeneity: } \mathrm{Tau}^{2}=0.01 ; \mathrm{Chi}^{2}=20.14, \mathrm{df}=3(\mathrm{P}=0.0002) ; \mathrm{l}^{2}=85 \% \\
\text { Test for overall effect: } Z=2.03(P=0.04)\end{array}$} & $\begin{array}{cc} & +1 \\
-0.2 & -0.1 \\
\text { Favours [DSF] }\end{array}$ & 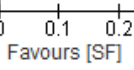 \\
\hline
\end{tabular}

Figure 8. The effect of DSF on HDL cholesterol (mmol/L).

\section{Discussion}

The findings of the systematic review and meta-analysis revealed that DSF was effective in lowering blood glucose (fasting blood glucose and glycated haemoglobin) compared with SF in patients with type 2 diabetes. However, the sensitivity analysis for the fasting blood glucose did not demonstrate a significant difference $(p>0.05)$ with the removal of the Pohl et al. [29,30] studies. In addition, there were no significant differences $(p>0.05)$ between the DSF and SF groups with respect to total cholesterol, LDL cholesterol and triglyceride (although a few studies reported significant differences with respect to triglyceride). Differences in the outcomes of studies in the systematic review were observed with respect to the effect of DSF on HDL cholesterol and the meta-analysis also showed significantly higher levels for the DSF group compared with the SF group. The high level of heterogeneity in the studies included in the meta-analyses may explain why the results of the meta-analysis and the sensitivity analysis were not consistent with respect to HDL cholesterol and fasting blood glucose.

The presence of low glycaemic index (GI) carbohydrate in the form of isomaltulose, the lower amount of carbohydrate and the higher protein content in the DSF may have contributed to the findings of this review [37]. In addition, the presence of mono-unsaturated fatty acids (MUFA) and the 
different amounts and types of fibre in the DSF compared with SF may be responsible for the observed differences in the fasting blood glucose, glycated haemoglobin and lipid profiles in both groups $[18,41]$. DSFs are usually higher in fat (40-50\% of energy with a significant portion of MUFA) and have a lower carbohydrate level (30-40\% of energy) and about $15 \%$ of energy is derived from fructose and soluble fibre [20]. DSFs contain carbohydrates with low GI such as non-hydrolysed starches, disaccharides, fibre and fructose in varying amounts which are aimed at controlling postprandial glucose [17-20]. In contrast, SFs are high in carbohydrate (about 50\%) and have low-moderate levels of lipids (about $30 \%$ ) and do not contain dietetic fibre [17]. A study by Hofman et al. [42] demonstrated that, in 12 enteral formulas examined, the GI ranged from 12 for DSFs up to 61 for SFs. The GI of food is a measure of how quickly the food is digested and the glucose reaches the blood stream [22,43]. Foods with high GI rapidly increase blood glucose and insulin responses after consumption [43,44]. The results from meta-analysis showed that the intake of a low GI diet was associated with reductions in blood glucose parameters [35,43]. In addition, high soluble fibre-containing foods can improve glycaemic control partly due to delayed absorption [36].

Therefore, DSFs may improve glycaemic control through delay in gastric emptying, delayed intestinal absorption of carbohydrate and lower glycaemic response [20]. In the study by Alish et al. [34], the blend of DSF was made up of low glycaemic and slowly digestible carbohydrates, resistant maltodextrin, isomaltulose, sucromalt and prebiotic fibres, including fructo-oligosaccharides. These constituents collectively produce a slow and consistent release of glucose into the blood stream [34]. Isomaltulose is a naturally occurring low GI slowly digestible carbohydrate [18]. The slower hydrolysation of isomaltulose during digestion may be responsible for the slower rise in blood glucose in patients with diabetes on DSF [18]. In addition, the higher protein content of the DSF may have contributed to the lowering of blood glucose parameters by delaying gastric emptying [18].

The use of high fat content, including MUFA, in the DSF may slow the transit time in the gastrointestinal tract and slow the absorption of sugars which could help improve glycaemic control [35,41]. Diets that are high in MUFA have been shown to increase HDL cholesterol and reduce other components of the lipid profiles [35,41]. HDL cholesterol is useful for reducing the risk of cardiovascular disease [35]. The result of the meta-analysis of the current review confirmed the positive role of DSF in increasing HDL cholesterol. However, the sensitivity tests did not demonstrate consistency in terms of the effect of DSF on HDL cholesterol, which could explain why researchers may be reluctant to recommend the use of high fat content in DSF due to the risk of alterations in lipid profiles [41]. This may also be due to the fact that there have been differences in the outcomes of studies on the effect of DSF on lipid profile [41].

\section{Limitation}

The limitation of this review was that only five studies were included in the meta-analysis. In particular, there were fewer studies included for lipid outcomes (three for several parameters) and there was substantial variability in the studies. Therefore, the differences between the meta-analysis and the sensitivity analysis in some of the parameters suggest that those results were not quite consistent, which may be due to the high level of heterogeneity of the studies. Therefore, more studies are needed to address this problem.

\section{Conclusions}

The results provide evidence to suggest that DSF is effective in controlling fasting blood glucose and glycated haemoglobin. In addition, DSF was effective in increasing HDL cholesterol but had no significant effect on other lipid parameters. However, our confidence in these findings would be increased by additional data from further studies. Additional research would also provide the opportunity to refine our understanding of the effect of DSF on cardiometabolic parameters.

Author Contributions: Conceptualization, O.O., S.M.W., T.T., R.C., X.-H.W.; methodology, O.O., S.M.W., T.T., R.C., X.-H.W.; validation, O.O., S.M.W., T.T., R.C., X.-H.W.; formal analysis, O.O. and reviewed by S.M.W., T.T., R.C., X.-H.W.; writing — original draft preparation, O.O.; writing—review and editing, O.O., S.M.W., T.T., R.C., X.-H.W. 
Funding: This research received no external funding.

Conflicts of Interest: The authors declare no conflicts of interest.

\section{References}

1. DeFronzo, R.A.; Ratner, R.E.; Han, J.; Kim, D.D.; Fineman, M.S.; Baron, A.D. Effects of exenatide (exendin-4) on glycemic control and weight over 30 weeks in metformin-treated patients with type 2 diabetes. Diabetes Care 2005, 28, 1092-1100. [CrossRef] [PubMed]

2. Jansink, R.; Braspenning, J.; Laurant, M.; Keizer, E.; Elwyn, G.; Weijden, T.D.; Grol, R. Minimal improvement of nurses' motivational interviewing skills in routine diabetes care one year after training: A cluster randomized trial. BMC Fam. Pract. 2013, 14, 44. [CrossRef] [PubMed]

3. National Collaborating Centre for Chronic Conditions (NCCCC). Type 2 Diabetes: National Clinical Guideline for Management in Primary and Secondary Care (Update); Royal College of Physicians: London, UK, 2008.

4. Public Health England. 3.8 Million People in England Now Have Diabetes. 2016. Available online: https://www.gov.uk/government/news/38-million-people-in-england-now-have-diabetes (accessed on 1 February 2019).

5. National Health Service (NHS) Digital and Healthcare Quality Improvement Partnership. National diabetes audit, 2015-2016 Report 1: Care Processes and Treatment Targets. 2017. Available online: http://www.content. digital.nhs.uk/catalogue/PUB23241/nati-diab-rep1-audi-2015-16.pdf (accessed on 1 February 2019).

6. Diabetes UK. State of the Nation Report. 2015. Available online: https://www.diabetes.org.uk/Documents/ About\%20Us/What\%20we\%20say/State\%20of\%20the\%20nation\%202014.pdf (accessed on 1 February 2019).

7. Holman, N.; Young, B.; Gadsby, R. What is the current prevalence of diagnosed and yet to be diagnosed diabetes in the UK. Diabetes Med. 2014, 31, 510-511. [CrossRef] [PubMed]

8. Diabetes UK. Diabetes in the UK 2012: Key Statistics on Diabetes. 2012. Available online: http://tinyurl.com/ owcyr7b (accessed on 1 February 2019).

9. Holmes, C.; Dyer, P. Diabetes training for nurses: The effectiveness of an inpatient diabetes half-day workshop. J. Diabetes Nurs. 2013, 17, 86-94.

10. Pereira, D.A.; da Silva Campos Costa, N.M.; Lima Sousa, A.L.; Veiga Jardim, P.B.; de Oliveira Zanini, C.R. The effect of educational intervention on the disease knowledge of diabetes mellitus patients. Rev. Lat. Am. De Enferm. 2012, 20, 478-485. [CrossRef] [PubMed]

11. Wyness, L. Understanding the role of diet in type 2 diabetes prevention. Br. J. Commun. Nurs. 2009, 14, 374-379. [CrossRef] [PubMed]

12. Ojo, O. The role of nutrition and hydration in disease prevention and patient safety. Br. J. Nurs. 2017, 26, 1020-1022. [CrossRef] [PubMed]

13. Arinzon, Z.; Shabat, S.; Shuval, I.; Peisakh, A.; Berner, Y. Prevalence of diabetes mellitus in elderly patients received enteral nutrition long-term care service. Arch. Gerontol. Geriatr. 2008, 47, 383-393. [CrossRef]

14. Wong, V.W.; Manoharan, M.; Mak, M. Managing hyperglycaemia in patients with diabetes on enteral nutrition: The role of a specialized diabetes team. Eur. J. Clin. Nutr. 2014, 68, 1305-1308. [CrossRef]

15. Oyibo, S.; Sagi, S.; Home, C. Glycaemic control during enteral tube feeding in patients with diabetes who have had a stroke: A twice-daily insulin regimen. Pract. Diabetes 2012, 29, 135-139. [CrossRef]

16. Elia, M.; Ceriello, A.; Laube, H.; Sinclair, A.J.; Engfer, M.; Stratton, R.J. Enteral nutritional support and use of diabetes specific formulas for patients with diabetes. Diabetes Care 2005, 28, 2267-2279. [CrossRef] [PubMed]

17. Sanz-Paris, A.; Álvarez Hernández, J.; Ballesteros-Pomar, M.D.; Botella-Romero, F.; León-Sanz, M.; Martín-Palmero, Á.; Olveira, G. Evidence-based recommendations and expert consensus on enteral nutrition in the adult patient with diabetes mellitus or hyperglycemia. Nutrition 2017, 41, 58-67. [CrossRef] [PubMed]

18. Ceriello, A.; Lansink, M.; Rouws, C.H.F.C.; van Laere, K.M.J.; Frost, G.S. Administration of a new diabetes specific enteral formula results in an improved $24 \mathrm{~h}$ glucose profile in type 2 diabetic patients. Diabetes Res. Clin. Pract. 2009, 84, 259-266. [CrossRef] [PubMed]

19. Hise, M.E.; Fuhrman, M.P. The Effect of Diabetes Specific Enteral Formulae on Clinical and Glycaemic Indicators; Parrish, C.R., Ed.; Nutrition Issues in Gastroenterology Series 74; Shugar Publishing: New York, NY, USA, 2009; pp. 20-36. 
20. Buranapin, S.; Siangruangsang, S.; Chantapanich, V.; Hengjeerajarus, N. The comparative study of diabetic specific formula and standard formula on postprandial plasma glucose control in type $2 \mathrm{DM}$ patients. J. Med. Assoc. Thail. 2014, 97, 582-588.

21. McMahon, M.M.; Nystrom, E.; Braunschweig, C.; Miles, J.; Compher, C. The American Society of Parenteral and Enteral Nutrition (ASPEN) Clinical Guidelines: Nutrition support of adult patients with hyperglycaemia. J. Parenter. Enter. Nutr. 2013, 37, 23-36. [CrossRef] [PubMed]

22. Ojo $\mathrm{O}$ and Brooke $\mathrm{J}$ Evaluation of the role of enteral nutrition in managing patients with Diabetes: A systematic review. Nutrients 2014, 6, 5142-5152. [CrossRef]

23. Jones, S.; Honnor, M.; Castro, E.; Alsmadi, A. Management of people with diabetes receiving artificial nutrition: A review. J. Diabetes Nurs. 2017, 21, 179-183.

24. Moher, D.; Liberati, A.; Tetzlaff, J.; Altman, D.G.; Prisma, G. Preferred reporting items for systematic reviews and meta-analyses: The PRISMA statement. BMJ 2009, 339, b2535. [CrossRef]

25. Methley, A.M.; Campbell, S.; Chew-Graham, C.; McNally, R.; Cheraghi-Sohi, S. PICO, PICOS and SPIDER: A comparison study of specificity and sensitivity in three search tools for qualitative systematic reviews. BMC Health Serv. Res. 2014, 14, 579. [CrossRef]

26. Critical Appraisal Skills Programme (CASP) Case Control Study Checklist. Available online: http://docs. wixstatic.com/ugd/dded87_afbfc99848f64537a53826e1f5b30b5c.pdf (accessed on 29 January 2019).

27. Higgins, J.P.T.; Green, S. Cochrane Handbook for Systematic Reviews of Interventions; Wiley-Blackwell: Hoboken, NJ, USA, 2009.

28. The Nordic Cochrane Centre. Review Manager (RevMan). In Computer Program; Version 5.3; The Nordic Cochrane Centre, The Cochrane Collaboration: Copenhagen, Denmark, 2014.

29. Pohl, M.; Mayr, P.; Mertl-Roetzer, M.; Lauster, F.; Lerch, M.; Eriksen, J.; Haslbeck, M.; Rahlfs, V.W. Glycaemic control in type II diabetic tube-fed patients with a new enteral formula low in carbohydrates and high in monounsaturated fatty acids: A randomised controlled trial. Eur. J. Clin. Nutr. 2005, 59, 1221-1232. [CrossRef]

30. Pohl, M.; Mayr, P.; Mertl-Roetzer, M.; Lauster, F.; Haslbeck, M.; Hipper, B.; Steube, D.; Tietjen, M.; Eriksen, J.; Rahlfs, V.W. Glycemic control in patients with type 2 diabetes mellitus with a disease-specific enteral formula: Stage II of a randomized, controlled multicenter trial. J. Parenter. Enter. Nutr. 2009, 33, 37-49. [CrossRef] [PubMed]

31. Craig, L.D.; Nicholson, S.; Silverstone, F.A.; Kennedy, R.D.; Coble Voss, A.; Allison, S. Use of a reduced-carbohydrate, modified-fat enteral formula for improving metabolic control and clinical outcomes in long-term care residents with type 2 diabetes: Results of a pilot trial. Nutrition 1998, 14, 529-534. [CrossRef]

32. Lansink, M.; van Laere, K.M.; Vendrig, L.; Rutten, G.E. Lower postprandial glucose responses at baseline and after 4 weeks use of a diabetes-specific formula in diabetes type 2 patients. Diabetes Res. Clin. Pract. 2011, 93, 421-429. [CrossRef] [PubMed]

33. Vaisman, N.; Lansink, M.; Rouws, C.H.; van Laere, K.M.; Segal, R.; Niv, E.; Bowling, T.E.; Waitzberg, D.L.; Morleyf, J.E. Tube feeding with a diabetes-specific feed for 12 weeks improves glycaemic control in type 2 diabetes patients. Clin. Nutr. 2009, 28, 549-555. [CrossRef] [PubMed]

34. Alish, C.J.; Garvey, W.T.; Maki, K.C.; Sacks, G.S.; Hustead, D.S.; Hegazi, R.A.; Mustad, V.A. A diabetes-specific enteral formula improves glycemic variability in patients with type 2 diabetes. Diabetes Technol. Ther. 2010, 12, 419-425. [CrossRef] [PubMed]

35. Gulati, S.; Misra, A.; Nanda, K.; Pandey, R.M.; Garg, V.; Ganguly, S.; Cheung, L. Efficacy and tolerance of a diabetes specific formula in patients with type 2 diabetes mellitus: An open label, randomized, crossover study. Diabetes Metab. Syndr. Clin. Res. Rev. 2015, 9, 252-257. [CrossRef] [PubMed]

36. Hofman, Z.; Lansink, M.; Rouws, C.; van Drunen, J.D.E.; Kuipers, H. Diabetes specific tube feed results in improved glycaemic and triglyceridaemic control during $6 \mathrm{~h}$ continuous feeding in diabetes patients. e-SPEN 2007, 2, 44-50. [CrossRef]

37. Lansink, M.; Hofman, Z.; Genovese, S.; Rouws, C.H.F.C.; Ceriello, A. Improved Glucose Profile in Patients with Type 2 Diabetes with a New, High-Protein, Diabetes-Specific Tube Feed During 4 Hours of Continuous Feeding. JPEN J. Parenter. Enter. Nutr. 2017, 41, 968-975. [CrossRef] 
38. Mesejo, A.; Montejo-Gonzalez, J.C.; Vaquerizo-Alonso, C.; Lobo-Tamer, G.; Zabarte-Martinez, M.; Herrero-Meseguer, J.I.; Escirbano, J.A.; Malpica, A.B.; Lozano, F.M. Diabetes-specific enteral nutrition formula in hyperglycemic, mechanically ventilated, critically ill patients: A prospective, open-label, blind-randomized, multicenter study. Crit. Care 2015, 19, 390. [CrossRef]

39. Voss, A.C.; Maki, K.C.; Garvey, W.T.; Hustead, D.S.; Alish, C.; Fix, B.; Mustad, V.A. Effect of two carbohydrate-modified tube-feeding formulas on metabolic responses in patients with type 2 diabetes. Nutrition 2008, 24, 990-997. [CrossRef]

40. Vanschoonbeek, K.; Lansink, M.; van Laere, K.M.; Senden, J.M.; Verdijk, L.B.; van Loon, L.J. Slowly digestible carbohydrate sources can be used to attenuate the postprandial glycemic response to the ingestion of diabetes-specific enteral formulas. Diabetes Educ. 2009, 35, 631-640. [CrossRef] [PubMed]

41. Vahabzadeh, D.; Valizadeh Hasanloei, M.A.; Vahdat Shariatpanahi, Z. Effect of high-fat, low-carbohydrate enteral formula versus standard enteral formula in hyperglycemic critically ill patients: A randomized clinical trial. Int. J. Diabetes Dev. Ctries 2019, 39, 173-180. [CrossRef]

42. Hofman, Z.; van Drunen, J.D.E.; de Later, C.; Kuipers, H. The Glycaemic index of standard and diabetes specific enteral formulas. Asia Pac. J. Clin. Nutr. 2006, 15, 412-417. [PubMed]

43. Ojo, O.; Ojo, O.O.; Adebowale, F.; Wang, X.H. The Effect of Dietary Glycaemic Index on Glycaemia in Patients with Type 2 Diabetes: A Systematic Review and Meta-Analysis of Randomized Controlled Trials. Nutrients 2018, 10, 373. [CrossRef] [PubMed]

44. Chang, K.T.; Lampe, J.W.; Schwarz, Y.; Breymeyer, K.L.; Noar, K.A.; Song, X.; Neuhouser, M.L. Low Glycemic Load Experimental Diet More Satiating Than High Glycemic Load Diet. Nutr. Cancer 2012, 64, 666-673. [CrossRef] [PubMed]

(C) 2019 by the authors. Licensee MDPI, Basel, Switzerland. This article is an open access article distributed under the terms and conditions of the Creative Commons Attribution (CC BY) license (http://creativecommons.org/licenses/by/4.0/). 